\title{
Acute Fixation Vs Casting in Treatment of Fracture Base of Fifth Metatarsal Comparative Study
}

E.M.Ibrahim, E.M.Bayoumy and A.G.Saad

Orthopedic Surgery, Dept., Faculty of Medicine, Benha Univ., Benha, Egypt

E-mail: ahmed.gala1575@gmail.com

Abstract

Background: The understanding of the anatomy of the proximal portion of the fifth metatarsal is essential as it explains the injury process and cures of various kinds of fifth metatarsal fractures that influence the appropriate treatment decision. Work objective: Comparison of clinical and radiographic outcomes among conservation therapy and acute surgical therapy of the fifth-metatarsal fracture base. Methods and patients: Twenty patients have been treated with percutaneous fixation with a $4,0 \mathrm{~mm}$ cannula screw and 20 have been conservatively treated with a lower knee cast. The length of the injury to hospital admission varied from 1 to 3 days with an average of 2 days between September 2020 and May 2021. The average follow-up time was six months (range from 5 to 7 months). Findings: Our results revealed that for zone 2 and zone 3 the acute fixation is superior than casting with respect to the union time and the AOFAS score. Conclusion: Early screw fixing is a safe and efficient therapeutic technique for acute fifth metatarsal base fractures, particularly zones 2 and 3. Early operational therapy leads to before clinical union and enables patients to return to regular daily activities rather than to cast therapy, leading to a high delayed union rate.

Keywords: Acute Fixation, Casting, Fifth Metatarsal .

\section{Introduction}

In 1902 Sir Robert Jones initially reported the fracture of the proximal fifth metatarsal. Jones provided accounts from six patients (one person) who suffered from fractures without a direct effect in this region (his injury occurred while dancing). Fractures of the fifth metatarsal among radiologists are surprisingly contentious. "1" Therefore in a description of proximal fifth metatarsal fractures it is advisable to use correct categorization rather than the phrase 'jones fracture.' Lawrence and Botte described the most often used categorization in 1993. It differentiates three kinds of approximate 5th metatarsal fractures based on injury mechanism, location, treatment choices and forecasting. The most frequent fractures in Zone 1 provide excellent results with conservative treatment. Fields 2 and 3 are susceptible to delayed union and even non-union fractures. "In order to formulate an appropriate treatment strategy, Torg established additional classifications for fractures in zone 2 and 3." 3" Non-union and recurrent fractures of the proximal fifth metatarsal metaphysis (i.e., the Jones fractures) may create problems because of a high occurrence. Surgical therapy has traditionally been reserved for high-performance sportsmen. However, all patients should be carefully considered for the fractures at the base of the fifth metatarsal owing to their restricted vascular supply. "2"

\section{Aim of the Work}

Comparison of conservative therapy and acute surgical treatment of the fifth metatarsal fracture basis of clinical and radiological findings.

\section{Patients And Methods}

This is a comparative study between conservative and percutaneous screw fixation in the treatment of acute fracture base of fifth metatarsal in patients presenting to Benha University Hospital from September 2020 to May 2021.

Twenty patients were treated by percutaneous fixation using cannulated $4.0 \mathrm{~mm}$ screw and twenty patients were treated conservatively with below knee cast.

Our Patients are above the age of 18 and all of the are fit for surgery .Regarding the fracture ,they suffer from base of fifth metatarsal fracture which is recent within less than 14 days ,closed and isolated fracture.

We exclude patients who are under 18 years old or unfit for surgery or patients who are unable to weight bear prior to fracture .Also, we excluded patients who had old trauma which are more than 14 days, prior fracture in the base of fifth metatarsal ,open fractures and pathological fractures other than osteoporosis.

\subsection{Patient evaluation}

Clinical history was taken from the patient in the sort of name, sex, age, job, address and smoking habits .We asked about other associated illness like diabetes, hypertension and cardiac condition Patients were asked about the mechanism of injury as well and if there is any associated injuries.

Then, we examined our patients which include clinical and radiological examination .Regarding the clinical examination, we did a standard foot examination and observing any tenderness swelling ,limitation range of motion of ankle, skin condition , neurovascular examination and also examination for associated injuries was performed.

Regarding radiological evaluation all patients were evaluated by plain X-rays :AP, oblique and lateral views of the foot. Preoperative imaging was used to classify the patient's fractures and to plan surgery according to Dameron, Lawerence and Quill (1995) classification which classified base of fifth into 3 zones: Tuberosity avulsion fracture in zone I,fracture at 
the metaphyseal-diaphyseal junction in zone II and distal diaphyseal stress fractures in zone III.

\subsection{Cast group}

A below knee cast was applied and instructions that no weight bearing is allowed for the $1^{\text {st }}$ six to eight weeks .Cast was removed after 6 to 8 weeks and follow up X-rays were done to follow the union of the fracture and instructions to partial weight bearing as tolerated with crutches .If the patient suffered from a delayed union ,he was followed up after further 4 to 8 weeks till complete union.

\subsection{Operative group:}

The patients were assessed for fitness for surgery by clinical history, examination and routine preoperative laboratory investigation .Standard consent was taken from the patients

Regarding the implant, cannulated screw $4.00 \mathrm{~mm}$ with or without a washer was placed percutaneously under image intensifier perpendicular to fracture line, the length of the screw ranged from $45 \mathrm{~mm}$ to $60 \mathrm{~mm}$.

\subsection{Surgical technique}

All the patients were anaesthetized by spinal anesthesia. Patients were operated on a standard radiolucent orthopedic table, in supine position under image intensifier guidance.

\subsection{Postoperative care}

We followed up all the patients for postoperative pain, function, footwear requirement, walking distance, gait abnormality, alignment.

The patient remains in the hospital overnight, and prophylactic parenteral antibiotics are administered for the first 24 hours postoperatively.

Patients were instructed not to weight bear first two weeks and below knee slab was done post operatively for two weeks.After stich removal, they were instructed to partial weight bearing with crutches till 6 weeks. Then, the patient starts on full weightbearing without any aids. However, if we found any loosening over the screw or widening of the fracture gap, then we wait for another 2 weeks till full weightbearing. After 12 weeks there were no restrictions for the patient for running or contact sports.

The American Orthopedic Foot and Ankle Society (AOFAS): used as a clinical rating system to monitor improvement following injury.

\section{Results}

We followed up all the patients for pain, function, footwear requirement, walking distance, gait abnormality, alignment and radiological assessment for union.

\section{The operative group (group A)}

The sample size was 20 patients, all patients achieved full union .Complications were present in six patients. Two patient were complicated by superficial infection treated by antibiotics, two patient were complicated by tingling which disappeared with weight bearing and medical treatment and two patients were complicated by metal-ware irritation but the patient get used to it when started normal activity.

Our patients' ages started from 18 to over 60 years, we put each patient in its age group to compare results of each group in the same management there was a high incidence among two age groups aged 31 to 45 years and 46 to 60 years. less incidence in age group 18 to 30 and over 60 years

In the operative group, male patients were 11 patients and female patient were lower by being 9 patients.

The site of fractures were mainly zone 2 and zone 3 . However, patients who suffered from zone 2 fracture were 16 patients while patients who experienced zone 3 fracture were only 4 patients.

The time of union was correlated with the time of return to normal activity, painless movement of the foot and radiological assessment, Patients achieved union ranging from 6 to 9 weeks, with mean of 7.200 weeks and \pm SD of 0.951 .

Also, we compared union time to each age group and found that patients who aged 18 to 30 years had mean union time of 6.333 weeks with \pm SD of \pm 0.577 .Patient aged 31 to 45 years had there mean union time of 7.000 weeks with \pm SD of \pm 1.000 . Patient aged 46 to 60 years had there mean union time of 7.286 weeks with \pm SD of \pm 0.756 . Patient aged over 60 years had there mean union time of 8.333 weeks with \pm SD of 0.577 .

AOFAS score was documented for each group at 6 weeks and at the end of follow up. In group A, AOFAS score at 6 weeks ranged from 60 to 78 , mean was 70.650 with SD of \pm 4.738 . AOFAS of this group recorded a range of 80 to 96 at the end of follow up with mean of 87.850 and a SD of \pm 5.314 .

Then we compared each age group, Patients who aged 18 to 30 years had AOFAS score of 73.667 at 6 weeks with SD of \pm 3.512 , and the AOFAS score of at the end of follow up was 91.333 with SD of \pm 1.155 . Patients who aged 31 to 45 years had AOFAS score of 72.857 at 6 weeks with SD of \pm 2.734 and the AOFAS score of at the end of follow up was 92.000 with SD of \pm 3.266 . Patients who aged 46 to 60 years had AOFAS score of 70.571 at 6 weeks with SD of \pm 3.780 and the AOFAS score of at the end of follow up was 84.714 with SD of \pm 4.716 . Patients who aged over 60 years had AOFAS score of 62.667 at 6 weeks with SD of \pm 3.055 and the AOFAS score of at the end of follow up was 82.000 with SD of \pm 2.000 .

The Cast group (group B):

The sample size was 20 patients all patients achieved full union, 3 patient were complicated by sudeck's atrophy, 4 patients suffered from delayed weight-bearing and 6 patients were complicated by both sudek's atrophy and delayed weight-bearing.

Our patients' ages started from 18 to over 60 years, we put each patient in its age group to compare results of each group in the same management, and it is 
apparent that nearly similar incidence between the 4 age groups.

In this group, male patients were 14 patients and female patient were lower by being 6 patients.

The sites of fracture were mainly zone 2 and zone 3. However, patients who suffered from zone 2 fracture were 18 patients while patients who experienced zone 3 fracture were only 2 patients.

The time of union was correlated with the time of return to normal activity, painless movement of the foot and radiological assessment.

Patients achieved union ranging from 6 to 10 weeks, with mean of 7.3 weeks and $\mathbf{I S D}$ of 1.129 .

In this group, we compared union time to each age group. Patients who aged 18 to 30 years had mean union time of 9.750 weeks with SD of \pm 1.708 . Patient aged 31 to 45 years had there mean union time of 9.833 weeks with SD of \pm 1.472 . Patient aged 46 to 60 years had there mean union time of 12.800 weeks with SD of \pm 1.095 . Patient aged over 60 years had there mean union time of 14.400 weeks with SD of 1.673 .

In group B, AOFAS score ranged from 40 to 70 , it's mean was 54.550 with SD of \pm 8.976 . AOFAS of this group recorded a range of 60 to 88 at the end of follow up with mean of 75, 650 and a SD of \pm 7.782 .

Then we compared each age group, Patients who aged 18 to 30 years had AOFAS score of 65.500 at

6 weeks with SD of \pm 4.123 , and the AOFAS score of at the end of follow up was 84.500 with SD of \pm 3.000. Patients who aged 31 to 45 years had AOFAS score of 58.333 at 6 weeks with SD of \pm 3.445 and the AOFAS score of at the end of follow up was 79.167 with SD of \pm 2.563 . Patients who aged 46 to 60 years had AOFAS score of 50.400 at 6 weeks with SD of \pm 7.925 and the AOFAS score of at the end of follow up was 72.000 with SD of \pm 6.000 . Patients who aged over 60 years had AOFAS score of 45.400 at 6 weeks with SD of \pm 4.980 and the AOFAS score of at the end of follow up was 68.000 with SD of \pm 7.071 .

Then, we compared both operative and cast groups for the age, sex zone of fracture, time of union and AOFAS score regarding age, sex and zone of fracture, results were insignificant towards the first three parameters.

However, results were significant towards both time needed for complete union and AOFAS score at six weeks and at the end of follow up.

Regarding complete union, operative group was found to have a complete union in weeks at a mean of 7.2000 which is less than cast group by 4.200 weeks. Which prove that the results were significant towards operative group.

Also, the results were significant towards operative group in each age group.

Moreover, AOFAS score was significant in operative group than cast group as it was higher in operative group by 16.1 at 6 weeks and by 12.2 at the end of follow up.

The results were also significant towards operative group in each age group.

After calculating $\mathrm{P}$ value to compare both groups according to time of union, 6 weeks and end of follow up AOFAS Score. $\mathrm{P}$ value is significant $<0.001$ which is highly significant in these items.

Table (1) Comparing complete union in different age groups in operative and conservative management.

\begin{tabular}{lcccc}
\hline Age Groups & \multicolumn{2}{c}{$\begin{array}{c}\text { Complete union (Weeks) } \\
\text { Group A } \\
\text { Mean } \pm \text { SD }\end{array}$} & $\begin{array}{c}\text { Group B } \\
\text { Mean } \pm \text { SD }\end{array}$ & \multicolumn{2}{c}{ T-Test } \\
& $6.333 \pm 0.577$ & $9.750 \pm 1.708$ & -3.260 & $0.022^{*}$ \\
\hline $\mathbf{1 8 - 3 0}$ Years & $7.000 \pm 1.000$ & $9.833 \pm 1.472$ & -4.117 & $0.002^{*}$ \\
31-45 Years & $7.286 \pm 0.756$ & $12.800 \pm 1.095$ & -10.382 & $<0.001^{*}$ \\
46-60 Years & $8.333 \pm 0.577$ & $14.400 \pm 1.673$ & -5.907 & $0.001^{*}$ \\
\hline >60 Years & & & & \\
\hline
\end{tabular}

Table (2) Comparing AOFAS in 6 weeks and at the end of follow up in different age groups in operative and conservative management

\begin{tabular}{llcccc}
\hline \multirow{2}{*}{ AOFAS } & \multicolumn{2}{c}{ Group A } & \multicolumn{2}{c}{ Group B } & \multicolumn{2}{c}{ T-Test } \\
& & $\begin{array}{c}\text { Mean } \pm \text { SD } \\
\text { Mean } \pm \text { SD }\end{array}$ & t & P-value \\
\hline \multirow{4}{*}{ At 6 Weeks } & $\mathbf{1 8 - 3 0}$ Years & $73.667 \pm 3.512$ & $65.500 \pm 4.123$ & 2.749 & $0.040^{*}$ \\
& $\mathbf{3 1 - 4 5}$ Years & $72.857 \pm 2.734$ & $58.333 \pm 3.445$ & 8.482 & $<0.001^{*}$ \\
& 46-60 Years & $70.571 \pm 3.780$ & $50.400 \pm 7.925$ & 5.935 & $<0.001^{*}$ \\
At the end of follow up & $>$ 60 Years & $62.667 \pm 3.055$ & $45.400 \pm 4.980$ & 5.334 & $0.002^{*}$ \\
& 18-30 Years & $91.333 \pm 1.155$ & $84.500 \pm 3.000$ & 3.673 & $0.014^{*}$ \\
& $\mathbf{3 1 - 4 5}$ Years & $92.000 \pm 3.266$ & $79.167 \pm 2.563$ & 7.775 & $<0.001^{*}$ \\
& $\mathbf{4 6 - 6 0}$ Years & $84.714 \pm 4.716$ & $72.000 \pm 6.000$ & 4.122 & $0.002^{*}$ \\
& $>$ 60 Years & $82.000 \pm 2.000$ & $68.000 \pm 7.071$ & 3.256 & $0.017^{*}$ \\
\hline
\end{tabular}




\section{Discussion}

Fractures in the proximal fifth metatarsal (i.e. the Jones fracture) may be troublesome due to the high frequency of non-union and non-operatory recurrence. Surgical therapy has traditionally been reserved for high-performance sportsmen. However, owing to its restricted blood supply, fractures at the base of the fifth metatarsal should be considered carefully for all patients." 2"

The primary objective of this research was to determine if early screw fixation or conservative treatment is better and more efficient. In individuals who wish to return to regular activities early on, early screw fixation may be highly advised. Our population of patients was an active group of (18-69) years in the cast group and (19-69) years in the operating group. Patients were productive and engaged in both groups.

The minimum monitoring time was five months and a maximum of seven months.

We utilised D Cameron, Lawrence and Quill's radiography categorization.

Complications were observed in 6 individuals in the surgical group. 2 patients are complicated by superficial infection, 2 tingling complicates, and 2 metal irritation complicates. The cast group was complicated more than the surgical group, 3 patients were complicated by sudeck's atrophy, 4 patients were delayed and 6 were complicated by both sudek's atrophy as well as delayed weight bearing, totaling 13 difficult patients.

The operation group achieved success with average clinical union of 7.2 weeks and average union of 11.7 weeks for the cast group showing substantial outcomes for the surgical group.

At six weeks and at the conclusion of the followup, the AOFAS score was recorded for each group. In group A (operating group), the AOFAS score varied from 60 to 78 , with an average SD of $\pm 4,738$ of 70,650. At the conclusion of the follow-up AOFAS from that group reported a gamut of 80 to 96 with a mean of 87,850 and an SD of $\pm 5,314$. In group B on the other hand, the AOFAS score varied from 40 to 70 , meaning 54,550 SD $\pm 8,976$. At the conclusion of the follow-up AOFAS of this group reported a range of 60 to 88 with a mean of 75,650 and an SD of $\pm 7,782$.

This demonstrated that the AOFAS score was important in the operating group rather than cast group, because the AOFAS score was 16.1 at 6 weeks and 12.2 at the conclusion of the monitoring period.

Hongdong et al. have made a study similar to the research conducted from April 2013 to May 2015, in which 42 avulsion fracture patients from the fifth type 2 metatarsal basement in the Ekrol Division studied.

23 patients undergoed a thread hollow operation, 12 males, 11 females with an average age of $43.4 \pm 9.2$ years and 19 patients treated with plasters fixation, including 10 male and 9 female, with an average age of $45.7 \pm 8.9$ years and 13 male and 9 female patients with plaster fixation.

For both union and (AOFAS) union patients were monitored for an average of 18 months.
The surgical group had a fracture healing time of 9.3 \pm 2.0 weeks, with an AOFAS score of 84.1 \pm 3.2 , whereas that of the conservative therapy group had an average healing time of $12.2 \pm 2.2$ with an AOFAS score of $84.2 \pm 4.2$ points. This confirms that the operational therapy of zone 2 fifth metatarsal bone fracture is superior than the short-term treatment" "There was a mistake. 20. 20 "Our research had findings that are comparable to those conducted by Tiago et al., 34 people, but only received surgical therapy. On average, 97 days after surgery, with minimum 80 and maximum 130 days, radiographic fracture therapy was seen, while return to sport happened, on average, after 73 days after surgery. The majority of fractures were categorised as Torg II (59\%), followed by Torg III (23\%) and Torg I. (18 percent ). Surgical therapy only took place with screws in $74 \%$ of the lesions, and with bone grafting screws in 26\%. All 34 patients underwept AOFAS scored before and after operation, and exhibited substantial increases of $<0.0001$ from 42 to 99." 6. 6 "As suggested at the conclusion of our research, Le and Anderson also recommended that nonoperative treatment of zone II and zone 3 fractures for high-level athletes should extend their play owing to high levels of postponement of union, re-fracture and nonunion. Therefore, operating fixation with a partly threaded intramedullary screw is recommended for compression and intrinsic support. They believed that it was essential for the person to be asymptomatic and with protective changes in suitable sizes." 77 "Our research was similar with the Doan and Hunt study, which evaluated the conservative and operative treatment of the base of the fifth metatarsal fracture. She observed that the acute, non-operatively treated fractures had a union rate of approximately $75 \%$, whereas the intramedullary screw treated fractures had a union percentage of 96. " 88 "They also discovered that surgical intervention in the form of an intramedullary solid screw is an option. This is an essential factor for athletes who want to return to sport faster and who want the most solid and lasting structure." 88 "Some studies have been examined with our study in certain results, such as the study by Park et al. which studied the effect of weight bearings on the conservative and surgical management of fractures of the 5th metatarsal cord on 86 subjects classified in a surgical or non-operational treatment group and later in two groups; There was no significant difference between the surgical and non-operative therapy groups in pain or bone resorption. In total, the bone resorption of $22.5 \%$ of individuals in the late weight-bearing group was greater by $6.5 \%$ compared to that of the early weight-bearing group. Bone union in the last weight-bearing group was accomplished 1 week later than in the early weight-bearing group. Early weight bearings were thus thought to reduce bone resorption and perhaps to promote bone union." 9. 9 "Studies confirmed our research's primary objective as the study by Mologne reported in a Cheung and Lui paper, the study was the first randomised controlled trial comparing early intramedullary screw fixation to casting of fractures of type I. Of the 37 patients 
recruited (18 casting patients and 19 screw fastening patients), he found that non-operative therapy is quite low (44 percent), double the time for clinical union and reverting into sports. These findings were comparable to ours, and confirmed the significance of the Jones fracture acute operational management " 3 "

Wu et al. conducted a research comparable to our study which found that surgery was required in the case of young people or athletes with a 5th metatarsal avulsion base fracture $(2-3 \mathrm{~mm})$. Closed reduction by percutaneous cannulated screw fixation is an efficient means of treating the fifth avulsion fracture of the base metatarsal. It may not only reduce surgical stress, but also offers tight internal fixation to preserve metatarsal blood flow and prevent peripheral nerve damage. The therapeutic results were evaluated between minimally invasive and conservative surgery in this research. The AOFAS and VAS-FA were significantly better in the operative group after 6 months of treatment $(\mathrm{p}<0.05)$. "10" There are studies that conclude similarly to the research done by Julia Bowes and Richard Buckley, published in the World Journal of Orthopedics, that it is more complex to treat zone 2 and zone 3 fractures, since they are recognisable for a longer time. Nonoperatory treatment of zone 2 and zone 3 fractures involves immobilisation and non-weight support over 8 weeks, and in the case of zone 3 stress fractures would be more long-lasting. Diaphysical stress fractures of type I and acute zone 2 fractures may be treated without surgery. However, owing to quicker clinical recovery and return to sport, surgical treatment of the highly active group should be addressed. The postponed Zone 2 and Zone 3 unions may be operationally or not. Operational intervention in the active population is suggested. Non-unions of symptomatic zone 2 and zone 3 should be treated operatively." 5" On the other hand, the Monteban et al. research has concluded that both zone 1 and zone 2 are safely capable of treating proximal fifth metatarsal fractures with good patient outcome, less complications and reoperations, reduced healthcare costs and with no increased financial cost, which did not support our study, apart from mental health, which was significantly higher. 5" 5" 11. 11 "Bis et al. had some differences with our research, because it did not recommend surgical treatment of fifth metatarsal proximal fracture base but argued that for minimally displaced Type 1 fractures and Type 2 fractures, weight-bearing restrictions and maybe a follow-up period are not required. The research revealed, in fact, the good outcomes of these fractures which were handled conservatously via early loading in both groups, without any advantage of the cast in relation to the functional elasticized bandage. On the other hand, both groups had the poorest results for type-3 fractures and the delayed return to work and sports. showing resemblance to our research" 12. 12 "Watson et al. examined the optimum base of fifth metatarsal zone II fractures and found it to be mostly at the middle of the basis of the fifth metatarsal at the lateral cartilage edge.

\section{Conclusion}

Early screw fixation is a safe and efficient therapeutic technique for acute fifth metatarsal fractures, particularly zones 2 and 3 Jones. Early operational therapy leads to before clinical union and enables patients to return to regular daily activities rather than to cast therapy, leading to a high delayed union rate.

\section{Recommendation}

Acute fracture base of 5th Zone 2 and Zone 3 should, in order to achieve early union and weight losses and to prevent delayed union problems as a delayed weight bearing and sudek's atrophy, be treated surgically with a $4 \mathrm{~mm}$ percutaneous cannulated screw.

\section{References}

[1] G.Carr, AW.Grieve, I.Greaves. The Jones fracture of the fifth metatarsal. Trauma.vol. 12(1),pp. 51-4, 2010.

[2] JR.Clements. Complications of Metatarsal Fractures. InComplications in Foot and Ankle Surgery. Springer, Cham.vol. 125-131,pp. 2017.

[3] CN.Cheung, TH.Lui. Proximal fifth metatarsal fractures: anatomy, classification, treatment and complications. Archives of trauma research.vol.5(4),2016.

[4] MA.Hongdong, LU.Zhichao, D.Yin, Y.Han, M.Yang. Comparison of conservative treatment and full thread hollow screw for treatment of Ekrol 2 fracture of the fifth metatarsal bone. Chinese Journal of Orthopaedics.vol. 38(21),pp. 1307-13,2018 .

[5] J.Bowes, R.Buckley. Fifth metatarsal fractures and current treatment. World journal of orthopedics. Dec 18.vol.7(12),pp.793, 2016.

[6] T.Baumfeld, R.Fernandes Rezende, C.Nery, JP.Batista, D.Baumfeld. Fifth Metatarsal Fractures in Professional Soccer Players: Case Series. Foot \& ankle specialist..vol. 14,pp. 186-193,2020.

[7] M.Le, R.Anderson. Zone II and III fifth metatarsal fractures in athletes. Current reviews in musculoskeletal medicine.vol. 10(1),pp. 86-93, 2017.

[8] KC.Doan, KJ.Hunt. Jones Fractures. In Sports Injuries of the Foot and Ankle. Springer, Berlin, Heidelberg.vol.8,pp.249-258, 2019.

[9] JY. Park, HN.Kim, YS.Hyun, JS.Park, HJ.Kwon, SH.Kang, GL.Kim. Effect of weight- bearing in conservative and operative management of fractures of the base of the fifth metatarsal bone. BioMed research international..vol.8,pp.78-99, 2017.

[10] GB.Wu, B.Li, YF.Yang. Comparative study of surgical and conservative treatments for fifth metatarsal base avulsion fractures (type I) in young adults or athletes. Journal of Orthopaedic Surgery.vol.26(1),pp. 2309499017747128,2017. 
[11]P.Monteban, J.van Den Berg, J.van Hees, S.Nijs, H.Hoekstra. The outcome of proximal fifth metatarsal fractures: redefining treatment strategies. European Journal of Trauma and Emergency Surgery.vol.44(5),pp. 72734,2018.

[12]C.Biz, M.Zamperetti, A.Gasparella, M.Dalmau-Pastor, M.Corradin, de G.Guttry, P .Ruggieri. Early radiographic and clinical outcomes of minimally displaced proximal fifth metatarsal fractures: cast vs functional bandage. Muscles, ligaments and tendons journal.vol.7(3),pp.532,2017.

[13] GI.Watson, SC.Karnovsky, G.Konin, MC.Drakos. Optimal starting point for fifth metatarsal zone II fractures: a cadaveric study. Foot \& ankle international.vol. 38(7),pp.8027,2017 . 